\title{
Spinal epiduroscopy as an educational tool
}

New Jersey Pain Medicine for the Difficult and Failed Pain, Robert Wood Johnson University Hospital Rahway, New Jersey, USA

Young Kook Choi

\section{LETTERS TO EDITOR}

Spinal epiduroscopy as a diagnostic tool for the treatment of lower back and leg pain has been well described by Jo [1]. Since medical treatment is based on accurate diagnosis, the importance of the diagnostic role of epiduroscopy cannot be over-emphasized; however, the use of epiduroscopy has been limited due to technical difficulty and cost containment.

Currently, the clinical significance of diagnostic epiduroscopy is not widely understood, and epiduroscopy is mainly used for therapeutic purposes. Diagnostic information from epiduroscopy is beneficial in determining the underlying epidural condition, which is also useful for educational purposes. Epidural steroid injection is a common procedure performed routinely with an unsupported assumption of nerve root inflammation since there is no opportunity to see the virtual inflammatory condition of the epidural contents, and epidural injections applied in such a blind fashion may fail to resolve the inflammation and pain. Epiduroscopy can help physicians see the actual status of epidural inflammation and establish a proper treatment plan leading to successful pain relief.

Major causes of spinal pain are attributable to intra-spinal disorders such as protruded discs and degener- ative changes of the disc, bone, and joint causing nerve root compression and spinal stenosis; however, the correlation between pain and spinal pathology has been controversial [2]. It is not uncommon that patients experiencing pain do not exhibit coinciding disc changes on imaging studies, as these may just show minor disc bulging or no disc changes at all. Patients may also have unilateral leg pain with contralateral disc protrusion or bilateral leg pain with unilateral disc protrusion. Macnab analyzed 68 patients with a diagnosis of nerve root compression, who did not reveal disc abnormalities on surgical exploration, and reported that fifty patients showed degenerative changes of lateral and foraminal structures, while eighteen patients did not show any appreciable pathologic changes [3]. Evidently, there is a discrepancy between anatomical changes and pain, but in any anatomical circumstances the pain can be derived from an inflammatory condition of the epidural contents, which are not shown on imaging studies.

Inflammation is initiated locally at the epidural and/or foraminal site, where the epidural contents are irritated biomechanically by the surrounding disc, bone, and joint structures. Depending on the location and magnitude of the inflammation, pain symptoms may vary. Locally developed inflammation grows beyond the primary lesion into

Received September 19, 2017. Revised October 1, 2017. Accepted October 12, 2017.

Correspondence to: Young Kook Choi

New Jersey Pain Medicine for the Difficult and Failed Pain, Robert Wood Johnson University Hospital Rahway, 1581 Lincoln Highway, Edison, New Jersey 08817, USA

Tel: + 1-732-754-0369, Fax: +1-855-492-5768, E-mail: choiyomd@hotmail.com

(c) This is an open-access article distributed under the terms of the Creative Commons Attribution Non-Commercial License (http:// creativecommons.org/licenses/by-nc/4.0/), which permits unrestricted non-commercial use, distribution, and reproduction in any medium, provided the original work is properly cited.

Copyright (c) The Korean Pain Society, 2018 
the neighboring tissue, and the pain symptoms may change accordingly. As the inflammation extends further to the other side of the epidural space across the midline, unilateral back and leg pain becomes bilateral.

Fibrotic tissue is also generated with inflammatory reaction and adheres to the inflamed epidural and foraminal contents gradually, which forms an inflammation fibrosis complex, sealing off the epidural and foraminal spaces around the primary lesion [4]. Subsequently, a closed cavity can be formed, which may interfere with the spread of injected steroid medications into the primary lesion.

Choi et al. [5] performed epiduroscopy on 24 patients, including 20 patients with previous surgeries and $4 \mathrm{pa}-$ tients without previous surgery, who failed to respond to conservative therapy and epidural steroid injections, and epiduroscopic findings showed extensive epidural inflammation with fibrosis beyond the specific dermatomal level of pain in all patients. All patients required a release of dense fibrotic tissue to resolve the inflammation at the primary lesion, as well as the surrounding inflammation. It is necessary to know the ongoing status of the epidural condition in order to achieve complete pain relief.

At this juncture, epiduroscopy is the only way to show epidural inflammation directly. Epiduroscopy was first introduced to investigate the epidural space in 1985 and has been applied to release fibrotic adhesion and deliver steroid medications precisely at the inflamed nerve root area under direct visualization [6]. Basically, epiduroscopy shows the real world of lumbosacral epiduropathy, including the causes of various types of spinal pain. The location, dis-
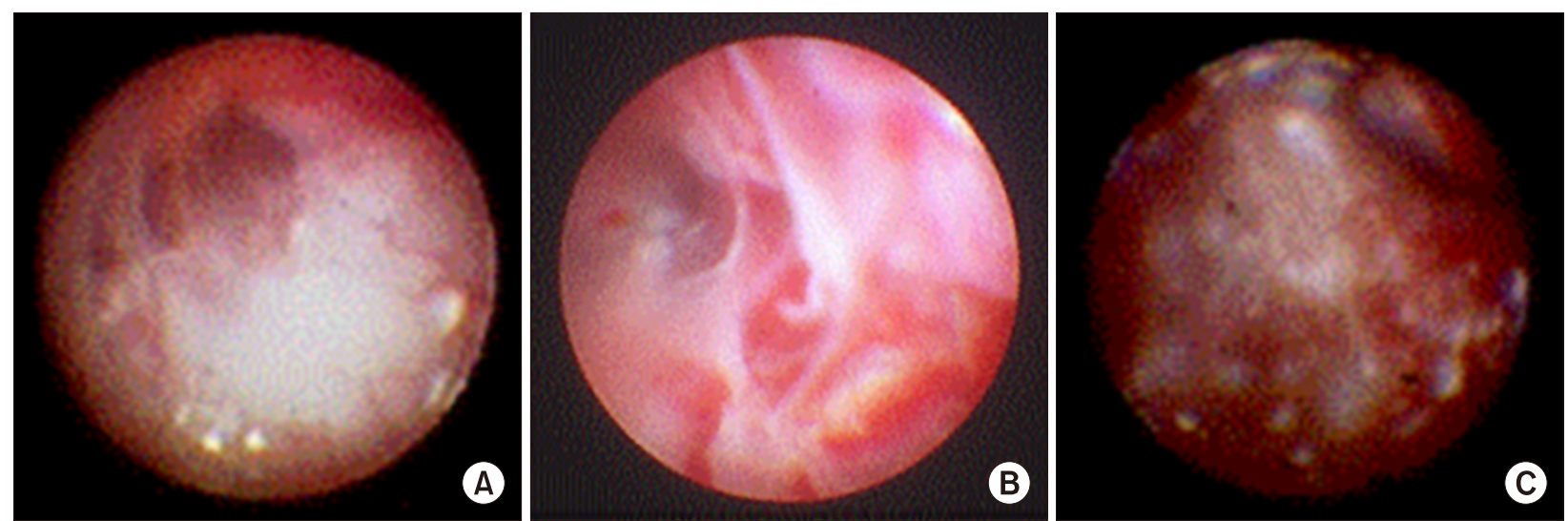

Fig. 1. Inflammation of epidural space. Mild inflammation locally around the dura is seen in (A). Moderate inflammation in the regional area around the dura with increased congestion and fibrosis is seen in (B). Extensive inflammation with granulation and fibrosis is seen in (C).
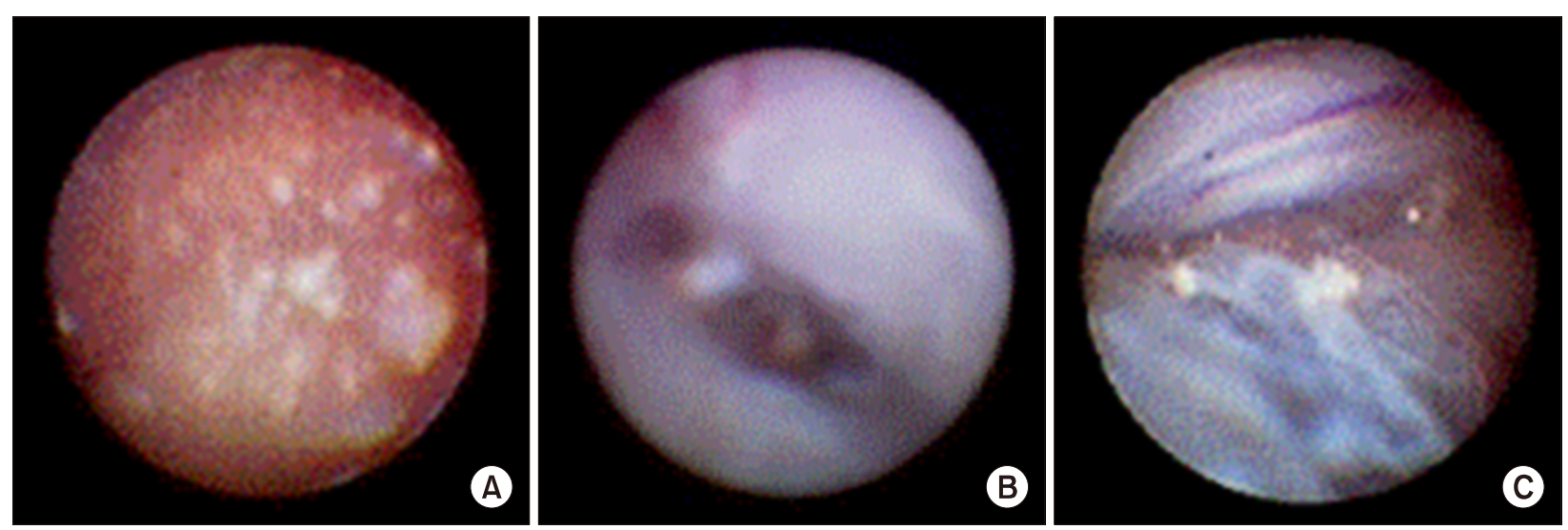

Fig. 2. Inflammation fibrosis complex of epidural space. Epidural space is filled with gelatinous exudates and fibrosis in (A), membranous fibrosis in (B), and fibrotic bands and meshes in (C). 
tribution, amount of inflammation (Fig. 1), fibrosis appearing strands, gelatins, bands, meshes, or membranes can be determined, and the extent of inflammation in the fibrosis complex can be graded as localized, regional, or extensive (Fig. 2).

Physicians can confirm actual pathologic conditions causing pain, while the pain perception represents an implicit psychological domain and various epidural conditions unresponsive to epidural steroid injections can be documented.

Collected diagnostic data are organized to review the status of the inflammatory condition at different locations, and its concordant pain symptoms, and a treatment paradigm with respect to specific pain problems, can be developed. Subsequently, an educational program is integrated into the curriculum for those who are in training systems to learn the basics of epidural pathology. As such, epiduroscopy can be used as an efficient diagnostic and educational tool.

\section{REFERENCES}

1. Jo DH. Epiduroscopy as a diagnostic tool for the lower back pain and/or leg pain. Korean J Pain 2016; 29: 151-2.

2. Videman T, Battié MC, Gibbons LE, Maravilla K, Manninen $\mathrm{H}$, Kaprio J. Associations between back pain history and lumbar MRI findings. Spine (Phila Pa 1976) 2003; 28: 582-8.

3. Macnab I. Negative disc exploration. An analysis of the causes of nerve-root involvement in sixty-eight patients. J Bone Joint Surg Am 1971; 53: 891-903.

4. Richardson J, McGurgan P, Cheema S, Prasad R, Gupta S. Spinal endoscopy in chronic low back pain with radiculopathy. A prospective case series. Anaesthesia 2001; 56: 454-60.

5. Choi YK, Tan MH, Barbella JD, Grubb WR. Epiduroscopic analysis of persistent back and leg pain and the efficacy of epiduroscopy in the treatment of failed back pain synsdrome. American Society of Anesthesiologists Annual Meeting 2000; 2000 Oct 14-18; San Fransisco, CA. Schaumburg, IL: American Society of Anesthesiologists; 2000. Abstr 937.

6. Saberski LR, Kitahata LM. Direct visualization of the lumbosacral epidural space through the sacral hiatus. Anesth Analg 1995; 80: 839-40. 